\section{Cetoacidosis diabética euglicémica asociada a inhibidor de cotransportador de sodio glucosa Tipo 2 en paciente con diabetes Mellitus Tipo 1}

\author{
VICTORIA NOVIK A. ${ }^{1}$, CLAUDIA GARCÍA B. ${ }^{2}$, DANIELA OLIVA I. ${ }^{3}$
}

\section{Diabetic ketoacidosis with mild hyperglycemia associated with the use of canagliflozin. Report of one case with type 1 diabetes}

Diabetic ketoacidosis with mild hyperglycemia is a major complication of sodium-glucose cotransporter 2 inhibitors. Although its use is not approved for patients with type 1 diabetes mellitus, the drug is often prescribed with the hope of optimizing metabolic control. We report a 20 years old female with hypothyroidism and type 1 diabetes consulting for vomiting and abdominal pain. The patient had used canagliflozin during the two previous months. Laboratory showed a blood glucose of $200 \mathrm{mg} / \mathrm{dl}$, a severe metabolic acidosis ( $p H 7.1$ ) and ketonemia. The patient was successfully treated in the intensive care unit.

(Rev Med Chile 2017; 145: 393-396)

Key words: Diabetes Mellitus, Type 1; Diabetic Ketoacidosis; Glycosuria; Hyperglycemia.
${ }^{1}$ Endocrinóloga, Diabetóloga. Profesor Adjunto, Universidad de Valparaíso, Chile. Profesor Asociado, Universidad Andrés Bello, sede Viña del Mar, Chile. ${ }^{2}$ Post becada Medicina Interna, Universidad de Valparaíso, Chile. ${ }^{3}$ Médico internista Hospital San Martín de Quillota, Chile.

Fuentes de apoyo financiero: no Conflicto de intereses: ninguno

Recibido el 8 de agosto de 2016, aceptado el 5 de diciembre de 2016.

Correspondencia:

Dra. Victoria Novik Assael

Asturias 1750 casa 6 Viña del

Mar Alto.

victorianovik@gmail.com

\section{L} os inhibidores del cotransportador de sodio-glucosa tipo 2 (iSGLT2)son antidiabéticos orales, que actúan a través de un mecanismo independiente de la secreción de insulina, mediante la disminución de reabsorción renal de glucosa en el túbulo contorneado proximal, obteniendo así la reducción de la glicemia y de su variabilidad, con baja incidencia de hipoglicemias ${ }^{1}$. Desde su aprobación para el uso en pacientes con diabetes mellitus tipo 2(DM2) por la Agencia de Drogas y Alimentos de EUA (Food and Drug Administration, FDA) en el año 2013, han sido reportados varios casos de cetoacidosis diabética (CAD), destacando la presentación atípica con glicemias no tan elevadas, incluso inferiores a $200 \mathrm{mg} / \mathrm{dl}^{2-3}$.

A pesar de que estos fármacos no están aprobados para ser usados en diabetes mellitus tipo 1 (DM1), se están prescribiendo actualmente debido a que su mecanismo de acción glucosúrico sería una alternativa factible para mejorar el control glicémico, reducir dosis de insulina y promover la pérdida de peso en pacientes con mal control metabólico ${ }^{4}$. Recordemos que hasta el $68 \%$ de los pacientes adultos con DM1 actualmente presentan sobrepeso u obesidad ${ }^{5}$. Un estudio reciente hecho en EEUU evidencia que hasta $47,8 \%$ de tales pacientes serían obesos ${ }^{6}$, una realidad que también se ha observado en nuestro país ${ }^{7}$.

A continuación reportamos un caso inusual de CAD en paciente DM1en que la hiperglicemia leve, asociada a glucosuria inapropiadamente alta hizo sospechar el uso de iSGLT2 que la paciente no había reportado en forma espontánea. Este sería el primer caso reportado en Chile.

\section{Caso clínico}

Paciente mujer de 30 años, con antecedentes de hipotiroidismo y DM1 desde los 8 años de edad. En su último control tres meses antes, su hemoglobina 
glicosilada (HbAlc) fue de 8,5\%. Consultó por cuadro de 24 horas de evolución con vómitos en siete ocasiones y dolor abdominal, sin diarrea ni fiebre. Debido a que no estaba ingiriendo alimentos en cantidad adecuada, la paciente omitió la dosis de insulina el mismo día de la consulta.

Dirigidamente, la paciente no refiere haber estado en un ayuno prolongado, la ingesta de alcohol ni en riesgo de embarazo previo al inicio de los síntomas.

$\mathrm{Al}$ ingreso, la paciente se encontraba vigil, $\mathrm{y}$ al examen con signos de leve déficit de volumen extra celular (VEC). Su Índice de masa corporal era 29 $\mathrm{Kg} / \mathrm{m}^{2}$. En sus exámenes de laboratorio al ingreso, destacaba: leucocitosis de $26.000 \mathrm{x} \mathrm{mm}^{3}$, glicemia $200 \mathrm{mg} / \mathrm{dl}$, gases arteriales con acidosis metabólica severa ( $\mathrm{pH} 7,1$ y bicarbonato de $7 \mathrm{meq} / \mathrm{L})$, cetonemia ++++++ (no se dispone de medición cuantitativa), cetonuria +++ , glucosuria. La proteína c reactiva, función renal, amilasemia y los electrolitos plasmáticos estaban dentro de límites normales.Una tomografía axial computarizada de abdomen y pelvis resultó sin alteraciones.

Ingresó a la Unidad de Cuidados Intensivos (UCI), iniciándose hidratación parenteral e insulina cristalina endovenosa, en infusión continua. Evolucionó favorablemente. Dada la presencia de una Cetoacidosis diabética (CAD) sin un desencadenante claro y la glucosuria inapropiadamente alta para tal nivel de hiperglicemia, se reinterroga a la paciente acerca del uso de otros medicamentos; entonces refiere haber iniciado canaglifozina dos meses previo al cuadro. Una vez estabilizada, se reajustó esquema de insulina y fue dada de alta al cuarto día de hospitalización.

Se realizó la notificación al Instituto de Salud Pública y se informó al Laboratorio Janssen Chile, constituyéndose en el primer caso descrito de CAD con el uso de estos medicamentos en nuestro país.

\section{Discusión}

Se han reportado varios casos de CAD en usuarios de iSGLT2, la gran mayoría en pacientes con DM2 $2^{8-9-10}$, que motivaron la publicación de un reporte de seguridad de la FDA en el año 2015 advirtiendo un mayor riesgo de CAD con hiperglicemia leve en pacientes en tratamiento con inhibidores deSGLT2, que se ha denominado CAD "euglicémica"11.
Esta disociación entre glicemia y glucosuria, en el contexto clínico de nuestra paciente con DM1, mal control metabólico y sobrepeso ${ }^{12}$ fue lo que llevó a sospechar en el uso de algún fármaco iSGLT2.

La CAD "euglicémica" se ha visto asociada a factores desencadenantes como infecciones intercurrentes, dieta baja en carbohidratos, reducción en la ingesta calórica total y uso de alcohol. También se ha relacionado con disminución u omisión de las dosis de insulinoterapia, o déficit de VEC por vómitos y falta de ingesta, tal como se observó en el presente caso.

Es importante recordar que la mayoría de los pacientes con DM1 en tratamiento exclusivo con insulina no cumple con las metas de HbAlc y presentan frecuentes episodios de hipoglicemia, por lo que se han buscado nuevas estrategias farmacológicas. La asociación de insulina con iSGLT2 han mostrado resultados prometedores en términos de reducción de peso, HbAlc,y de la dosis de insulina. Algunos trabajos también han demostrado una menor incidencia de hipoglicemia. Sin embargo, tales estudios son de corta duración, y con un escaso número de pacientes participantes ${ }^{12-14}$. La racionalidad del uso de este grupo de fármacos en pacientes diabéticos radica en que no requieren indemnidad de la célula beta pancreática ${ }^{4}$.

Los pacientes con DM1 usuarios de iSGLT2 presentan mayor riesgo de desarrollar cetoacidosis “euglicémicas" que los pacientes con DM 215. Su fisiopatología exacta no se conoce y está bajo investigación ${ }^{16,17}$, sin embargo, el mecanismo propuesto sería la asociación de deshidratación y glucosuria intensas, que conducirían a la cetosis por inanición, agravada por el aumento en los niveles de glucagón y ácido láctico ${ }^{18}$.

Aunque los iSGLT2 no han sido aprobados aún para el uso en DM1, la Asociación Americana de Endocrinólogos Clínicos y el Colegio Americano de Endocrinología, promueve la continuación de los estudios en curso. Cabe destacar un estudio de 18 semanas de duración, aleatorio, doble ciego en pacientes DM1 usuarios de insulina, en que se observó una incidencia de 4,3\% y $6 \%$ de CAD grave asociado al uso de canaglifozina de 100 y 300 $\mathrm{mg}$, respectivamente. Ninguno de los pacientes del grupo placebo presentó este tipo de complicación. Se concluyó entonces que canaglifozina se asocia a mayor incidencia de episodios graves de CAD en este tipo de pacientes ${ }^{19}$. 
Para minimizar el riesgo de la CAD asociado con esta familia terapéutica, se recomienda suspenderlos al menos $24 \mathrm{~h}$ antes de una cirugía electiva, previo a actividad física intensa, y procedimientos invasivos que impliquen un ayuno prolongado ${ }^{20}$.

$\mathrm{Al}$ igual que en nuestro caso,estos episodios se presentan con un menor nivel de glicemia que la CAD habitual, por lo que es necesario que los pacientes en tratamiento con inhibidores de SGLT2 que presenten compromiso del estado general, náuseas o vómitos, verifiquen sus niveles de cetonas ya sea en sangre o en orina y que si éstas están presentes, suspendan inmediatamente el fármaco y acudan al Servicio de Urgencia.

Se recomienda, además, que el médico que prescriba estos fármacos esté familiarizado con las indicaciones y advertencias actuales y eduque activamente al paciente al respecto, para evitar en lo posible una complicación no exenta de morbimortalidad.

En conclusión, nos parece relevante sospechar una CAD en pacientes diabéticos usuarios de iSGLT2 sin esperar una hiperglicemia importante, especialmente si es que cursa en forma concomitante con alguna situación de déficit de VEC asociada a falta de ingesta de carbohidratos u omisión de la dosis de insulina. Es importante respetar las recomendaciones de la FDA que actualmente no aprueba este fármaco en pacientes con DM1.

Agradecimientos: A las internas de la Universidad Andrés Bello sede Viña del Mar, Catalina Barros, Catalina Acuña y Giuliana Lavagnino, por el entusiasmo e interés durante la vivencia del caso clínico. Y a los revisores externos, anónimos, de la Revista, que con sus sugerencias han contribuido a la mejoría del manuscrito.

\section{Referencias}

1. Vivian EM. Sodium-glucose co-transporter 2 (SGLT2) inhibitors: a growing class of antidiabetic agents. Drugs in Context 2014; 3: 212-64.

2. Peters A, Buschur E, Buse J, Cohan P, Diner J, Hirsch I. Euglycemic Diabetic Ketoacidosis: A Potential Complication of Treatment With Sodium-Glucose Cotransporter 2 Inhibition. Diabetes Care 2015; 38: 1687-93.

3. Erondu N, Desai M, Ways K, Meininger G. Diabetic Ketoacidosis and Related Events in the Canagliflozin Type 2 Diabetes Clinical Program. Diabetes Care 2015;
38: $1680-86$.

4. Henry R, Thakkar P, Tong C, Polidori D, Alba M. Efficacy and Safety of Canagliflozin, a Sodium-Glucose Cotransporter 2 Inhibitor, as Add-on to Insulin in Patients With Type 1 Diabetes. Diabetes Care 2015; 38: 2258-65.

5. Miller KM, Foster NC, Beck RW, Bergenstal RM, Dubose SN, dimeglio LA, et al. Current state of type 1 diabetes treatment in the U.S.: updated data from the T1D Exchange clinic registry.Diabetes Care. 2015 Jun; 38: 971-8.

6. Analysis of physician electronic health records in the US from 2009-2011. Journal of Diabetes and Its Complications 2016; 30: 212-20.

7. Baez M, Novik V, Alegria F, Cardemil F, Riveros R, Bofill L. Síndrome metabólico en un grupo de pacientes diabéticos tipo 1. ¿Una nueva variedad de diabetes?. Rev Med Chile 2009; 137: 888-93.

8. Andrews TJ, Cox RD, Parker C, Kolb J. Euglycemic Diabetic Ketoacidosis with Elevated Acetone in a Patient Taking a Sodium-Glucose Cotransporter-2 (SGLT2) Inhibitor. J Emerg Med 2016 Oct 4. Pii: S07364679(16)30533-9.

9. Roach P, SkierczynskiP. Euglycemic Diabetic Ketoacidosis in a Patient With Type 2 Diabetes After Treatment With Empagliflozin. Diabetes Care. 2016 Jan;39(1): e3e3.

10. Jazi M, Porfiris G. Euglycemic diabetic ketoacidosis in type 2 diabetes treated with a sodium-glucose cotransporter-2 inhibitor. Can Fam Physician 2016 Sep; 62: 722-4.

11. FDA. Comunicado de la FDA sobre la seguridad de los medicamentos: La FDA advierte que el uso de inhibidores del SGLT2 para la diabetes puede provocar una grave concentración de ácido en la sangre. Disponible en:http://www.fda.gov/Drugs/drugsafety/ucm447209. htm

12. Bell D. Case Reports That Illustrate the Efficacy of SGLT2 Inhibitors in the Type 1 Diabetic Patient. Case Reports in Endocrinology 2015; (2) 1-4.

13. Pieber TR, Famulla S, Eilbracht J, Cescutti J, Soleymanlou N, Johansen OE, et al. Empagliflozin as adjunct to insulin in patients with type 1 diabetes: a 4-week, randomized, placebo-controlled trial (EASE-1).Diabetes Obes Metab 2015; 17(10): 928-35.

14. Sands AT, Zambrowicz BP, Rosenstock J, Lapuerta P, Bode BW, Garg SK, et al. Sotagliflozin, a Dual SGLT1 and SGLT2 Inhibitor, as Adjunct Therapy to Insulin in Type 1 Diabetes. Diabetes Care 2015; 38 (7): 1181-8.

15. Abbas K, Umpierrez G, Miles J, Fisher J. Hyperglycemic Crises in Adult Patients With Diabetes. Diabetes Care 2009; 32: 1335-43. 
16. Ogawa W, Sakaguchi K. Euglycemic diabetic ketoacidosis induced by SGLT2 inhibitors: possible mechanism and contributing factors. Journal of Diabetes Investigation 2016; 7: 135-8.

17. Taylor SI, Blau JE, Rother KI. SGLT2 Inhibitors May Predispose to Ketoacidosis. J Clin Endocrinol Metab 2015; 100(8): 2849-52.

18. Bode B, Garg S. The Emerging Role of Adjunctive Noninsulin Antihyperglycemic Therapy in the Mana- gement of Type 1. Endocr Pract. 2016; 22: 220-30.

19. Peters A, Henry R, Thakkar P, Tong C, Alba M. Diabetic Ketoacidosis With Canagliflozin, a Sodium-Glucose Cotransporter 2 Inhibitor, in Patients With Type 1 Diabetes. Diabetes Care 2016; 39: 532-38.

20. EMA makes recommendations to minimize ketoacidosis with SGLT2 inhibitors. Healthcare professionals should be aware of possible atypical cases. The Pharmaceutical Journal 2016; 296: 7886-8. 